\title{
The Evolving Role of Pre-pectoral ADM-assisted Approach in Implant-based Immediate Breast Reconstruction Following Conservative Mastectomy: An Overview of the Literature and Description of Technique
}

\author{
UMAR WAZIR and KEFAH MOKBEL
}

The London Breast Institute, Princess Grace Hospital, London, U.K.

\begin{abstract}
Background/Aim: The recent development of acellular dermal matrix (ADM) devices has enhanced implantbased breast reconstruction surgery following conservative mastectomy for therapeutic and risk-reducing purposes leading to improved aesthetics. In the traditional sub-pectoral approach, coverage of the implant is provided by the pectoral muscles superiorly and the ADM inferiorly. The need to eliminate breast animation, reduce post-operative dysfunctional pain and the risk of capsular contracture, have stimulated surgeons to investigate the feasibility of placing the implant over the pectoralis major muscle with complete coverage with ADM thus inventing a novel pre-pectoral approach. Materials and Methods: We reviewed the literature regarding this evolving technique of muscle sparing ADMassisted implant-based immediate breast reconstruction. Also, we describe our technique, and present pictures of the postoperative result. Results and Conclusion: The early reported results of the pre-pectoral breast reconstruction approach are encouraging and confirmed the potential benefits of eliminating breast animation and reducing postoperative pain. However, most of these studies had a small sample size $(<100$ patients) and were retrospective in nature with a limited follow-up duration and lack of data regarding the objective aesthetic assessment and oncological outcome. Nevertheless, suitable patients undergoing conservative mastectomy and implant-based reconstruction should be offered this option while further evaluation is being performed.
\end{abstract}

This article is freely accessible online.

Correspondence to: Prof. Kefah Mokbel, The London Breast Institute, Princess Grace Hospital, 42-52 Nottingham Place, London W1U 5NY, U.K. Tel: +44 02079082040, e-mail: kefahmokbel@hotmail.com

Key Words: Breast reconstruction, breast cancer, dermal matrix, pectoral muscle.
The recent development of acellular dermal matrix (ADM) devices has enhanced implant-based breast reconstruction surgery leading to improved aesthetics. In the traditional sub pectoral approach, coverage of the implant is provided by the pectoral muscles superiorly and the ADM inferiorly (1). There is currently a wide variety of ADM devices and the number of these biological matrices continues to increase. These matrices vary in their tissue of origin and processing procedures and as a result differ in their thickness, mechanical and physical characteristics, integration within the host and inflammatory reactions (1).

Our unit has had experience in the use of foetal bovinederived ADM marketed as SurgiMend ${ }^{\mathrm{TM}}$ (Integra Life Sciences, Plainsboro, NJ, USA) in implant-based breast reconstruction. We recently reviewed our practice and have gathered data in relation to patient outcomes. Our findings were favourable with regards to subjective patient satisfaction, aesthetic result and wound complications with their use $(1,2)$.

In this study, our experience is discussed in relation to the literature and our technique of prepectoral ADM-assisted implant-based immediate reconstruction following nipple sparing mastectomy using a fenestrated form of SurgiMend is presented. The fenestration technique we have employed enables coverage of greater surface areas than would otherwise been possible.

Surgical technique. In the following section, we describe the procedure as performed in a 35-year-old lady, who had neoadjuvant chemotherapy for right-sided disease. She had immediate reconstruction following nipple-sparing mastectomy.

This technique was undertaken in the context of nipplesparing mastectomy. This was performed via a circum-hemiareolar incision. Care should be taken to retain adequate thickness of the mastectomy flaps whilst ensuring that all the oncologically relative breast tissue is excised. 
After adequate haemostasis in the mastectomy bed is ensured, the pre-pectoral ADM-based pocket for the implant is fashioned. A meshed SurgiMend device was used, which is derived from foetal bovine material.

The ADM device was secured with $2 / 0$ polydioxanone sutures. First, the medial edge of ADM was sutured to the parasternal chest wall $1.5 \mathrm{~cm}$ from midline (Figure 1).

Then, the superior edge of the ADM device was secured to the underlying pectoralis major muscle just below the second rib (Figure 2).

The implant was then placed in the space between the ADM device and the pectoral muscles (Figure 3). After ensuring correct orientation of the implant and hemostasis, the inferior edge of the ADM device was secured to the inferior mammary fold (Figure 4).

After complete coverage of the implant by the ADM device is ensured (Figure 5), the skin was closed with subcuticular sutures of 3-0 monofilament polyglyconate.

The patient was highly satisfied with the outcome of the surgery. The skin healed well, and the aesthetic outcome was good (Figure 6). Significantly, there was no breast animation defect on arm raising (Figure 7).

\section{Discussion}

Initially, ADM devices were used to supplement the coverage provided by the pectoral muscles to an implant placed in a sub-pectoral pocket by covering the lower pole of the implant, thus complementing the pectoralis major muscle, in order to achieve complete coverage of the implant. This approach minimised the need to dissect other muscles, such as the serratus anterior leading to less morbidity due to post-operative pain and improved aesthetics with reduced visibility and palpability of the implant.

However, a further refinement in the use of ADM devices in implant-based breast reconstruction has recently led to the revival of the pre-pectoral placement of the implant, rather than the more conventional sub-pectoral position. The subpectoral approach is associated with breast animation, which can be a distressing symptom especially in physically-active women, and dysfunctional pain related to surgical dissection of the pectoralis major muscle. This approach was previously investigated but rejected due to the high rate of complications seen with the pre-pectoral approach in the preADM era.

However, the introduction of ADM devices, along with the increased use of fat transfer combined with skin- or nipple-sparing mastectomy (SSM; NSM) have revived the pre-pectoral approach as a viable alternative with complete implant coverage with ADM (3). The use of this approach is expected to avoid the issues pertaining to breast animation and post-operative dysfunctional pain which are inherent to sub-pectoral implant placement $(1,2)$ resulting in reduced

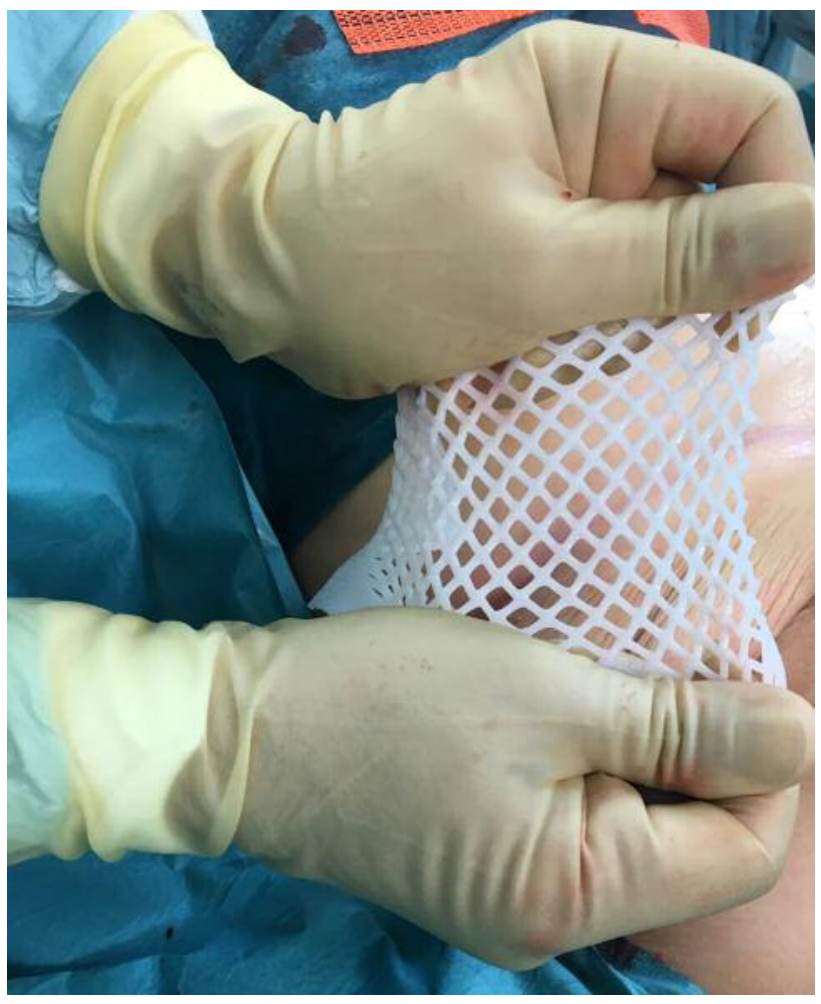

Figure 1. Preparation of the medial edge of ADM-based pouch. The medial edge of ADM is being sutured with PDS to the parasternal chest wall $1.5 \mathrm{~cm}$ from midline.

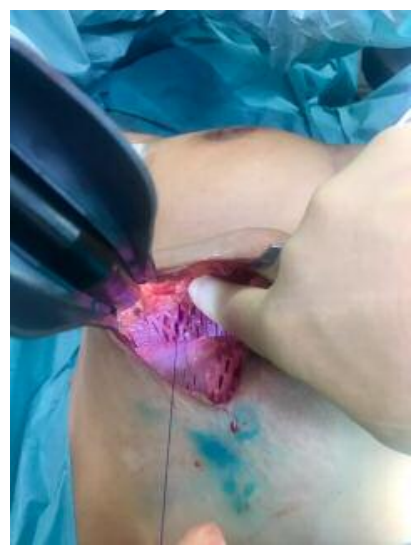

Figure 2. Preparation of the superior edge of ADM-based pouch. The superior edge of the Meshed ADM is being sutured with 2:0 PDS to the underlying pectoral muscle just above the second rib.

hospitalisation, need for physiotherapy and improved costeffectiveness. In particular, these new techniques have greatly facilitated risk-reducing mastectomies in genetically predisposed women (4). 


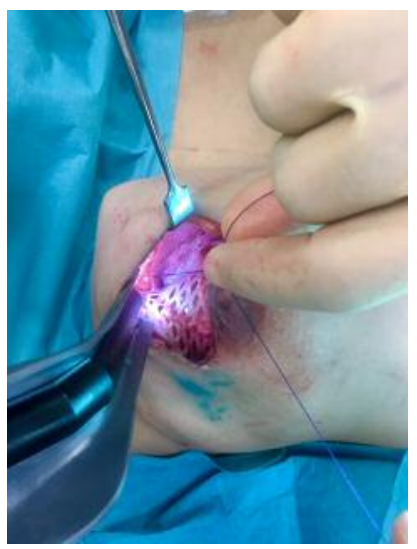

Figure 3. Placement of the implant in the pouch. The microtextured implant lies under the ADM over the pectoral muscle.

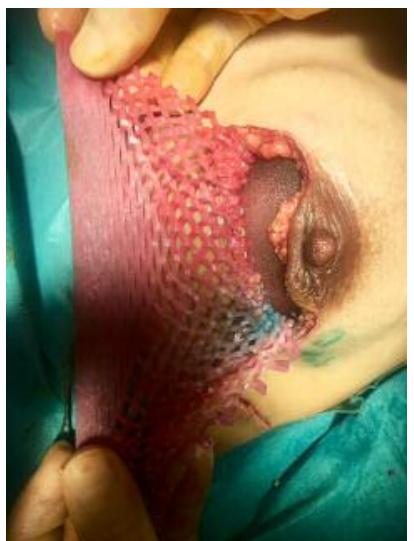

Figure 4. Preparation of the inferior edge of ADM-based pouch. The inferior edge of $A D M$ is sutured to the inframammary fold with 2:0 polydioxanone.

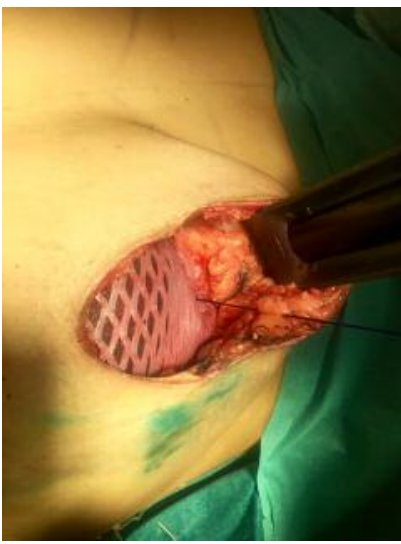

Figure 5. Anterior coverage of the implant with ADM. Meshed ADM provided complete anterior coverage of implant.

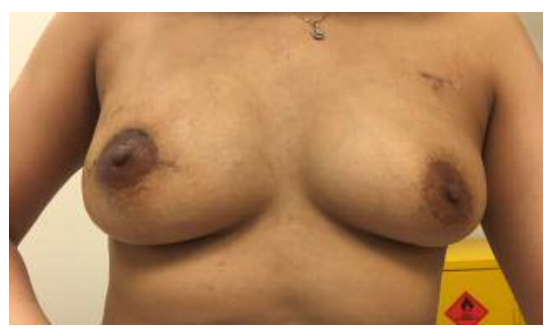

Figure 6. Early postoperative outcome 1 week after surgery. Anterior view, arms down.

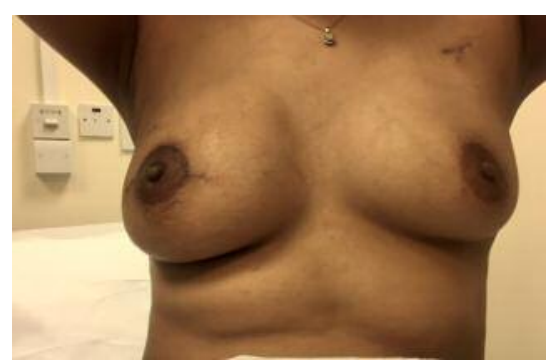

Figure 7. Early postoperative outcome 1 week after surgery: no animation when raising the arms. Anterior view, arms raise.

The ADM-based pre-pectoral approach can potentially result in improved aesthetics by facilitating surgeon's ability to fully control the aesthetic definition of the implant pocket. However, the muscle-sparing approach could potentially lead to an increased incidence of rippling and palpability and visibility of the implant in the upper pole of the reconstructed breast. Furthermore, it is not ideal for deeply located tumors that may extend to the deep margin. Therefore, careful patient selection with regards to comorbidities, oncological considerations (tumor extent and location, radiation therapy), anatomical parameters, accurate intra-operative assessment of mastectomy flap viability, thickness of the skin and subcutaneous fat are likely factors in achieving a successful outcome. These parameters require adequate assessment through objective aesthetic assessment in prospectively designed studies.

The technique can be used for both immediate direct implant-based reconstruction and two-staged prosthetic reconstruction. The number of subsequent procedures required to optimize the reconstruction, such as fat transfer, in order to reduce post-operative rippling, should be also considered when assessing cost effectiveness.

The pre-pectoral approach continues to increase in popularity and several authors have recently reported positive early results, using this muscle sparing ADM-assisted technique. However, 
most of these reports contain insufficient numbers of patients $(<100)$ and were retrospective in nature with a very short follow-up $(5,6)$. The high level of heterogeneity, the lack of a standardised technique and patient selection criteria, the type of ADM used represent further limitations compromising the quality of studies. Adequately powered prospective studies which would allow for robust conclusions to be drawn regarding aesthetic outcome, explanation rate, wound complications, subjective patient satisfaction, rate of capsular contracture and oncological outcome, are required for complete evaluation of this approach. The oncological outcome is an important consideration as surgeons enthusiastic about this technique could be subconsciously inclined to create thicker mastectomy flaps, which would improve the aesthetic outcome, but could compromise oncological outcome due to potentially leaving a greater amount of residual breast tissue or disease. Based on our experience, we believe that the final decision to proceed with pre-pectoral breast reconstruction can be only accurately made intra-operatively after carrying out meticulous skin sparing mastectomy through the correct plane of superficial fascia separating the subcutaneous fat from the underlying breast tissue (7). Furthermore, it is not currently known whether this technique could compromise the early detection of chest wall recurrence behind the implant, especially for tumours at an increased risk of chest wall recurrence, and whether magnetic resonance imaging (MRI) should be considered in such cases. Finally, the role of this evolving technique in the context of post-mastectomy radiation or prior radiotherapy should be also evaluated.

\section{Conclusion}

This new technique, as part of the breast reconstruction landscape, will continue to evolve being refined by current and future research. In addition to the benefits of preventing breast animations and reducing dysfunctional pain, it is likely that the addition of another biological covering to the interface between the implant and the host could significantly lower the rate of capsular contracture and possibly reduce the risk of anaplastic large cell lymphoma (ALCL) (8), which could represent a significant advantage of this approach. Furthermore, the absence of the mechanical force generated by pectoral muscle contractions could potentially reduce the risk of implant rupture.

\section{Conflicts of Interest}

The senior Author (KM) provides clinical and academic consultancy services to Q Medical Technologies.

\section{References}

1 Headon H, Kasem A, Manson A, Choy C, Carmichael AR and Mokbel K: Clinical outcome and patient satisfaction with the use of bovine-derived acellular dermal matrix (surgimend) in implant based immediate reconstruction following skin sparing mastectomy: A prospective observational study in a single centre. Surg Oncol 25(2): 104-110, 2016.

2 El Hage Chehade H, Headon H, Wazir U, Carmaichael AR, Choy C, Kasem A and Mokbel K: Nipple-sparing mastectomy using a hemi-periareolar incision with or without minimal medial-lateral extensions; clinical outcome and patient satisfaction: A single centre prospective observational study. Am J Surg 213(6): 1116-1124, 2017.

3 Wazir U, El Hage Chehade H, Headon H, Oteifa M, Kasem A and Mokbel K: Oncological safety of lipofilling in patients with breast cancer: A meta-analysis and update on clinical practice. Anticancer Res 36(9): 4521-4528, 2016.

4 Salhab M, Bismohun S and Mokbel K: Risk-reducing strategies for women carrying brcal/2 mutations with a focus on prophylactic surgery. BMC Womens Health 10: 28, 2010.

5 Sbitany H, Piper $M$ and Lentz $R$ : Prepectoral breast reconstruction: A safe alternative to submuscular prosthetic reconstruction following nipple-sparing mastectomy. Plast Reconstr Surg 140(3): 432-443, 2017.

6 Jones G, Yoo A, King V, Jao B, Wang H, Rammos C and Elwood E: Prepectoral immediate direct-to-implant breast reconstruction with anterior alloderm coverage. Plast Reconstr Surg 140(6S Prepectoral Breast Reconstruction): 31S-38S, 2017.

7 Cunnick GH and Mokbel K: Skin-sparing mastectomy. Am J Surg 188(1): 78-84, 2004.

8 Headon H, Kasem A and Mokbel K: Capsular contracture after breast augmentation: An update for clinical practice. Arch Plast Surg 42(5): 532-543, 2015. 